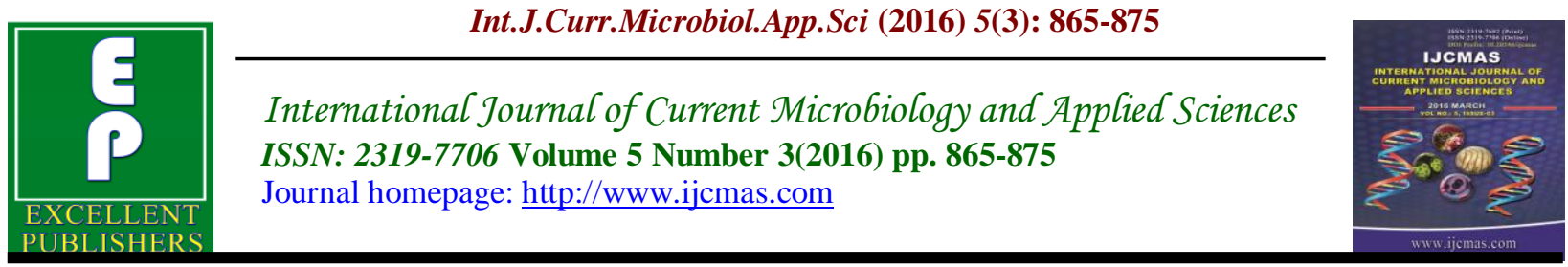

Original Research Article

http://dx.doi.org/10.20546/ijcmas.2016.503.100

\title{
Alterations of miR-320 Family Members as a Novel Diagnostic Biomarkers in Peripheral Blood of Schizophrenia Patients
}

\author{
Tihomir Iliev Vachev ${ }^{1,2 *}$, Nikolay Todorov Popov ${ }^{4}$, Vili Krasteva Stoyanova ${ }^{2,3}$, \\ Hristo Yordanov Ivanov ${ }^{2}$ and Danail Savov Minchev ${ }^{1}$ \\ ${ }^{1}$ Department of Plant Phisyology and Molecular Biology, University of Plovdiv "Paisii \\ Hilendarski”, 24 Tzar Assen Str., Plovdiv, Bulgaria \\ ${ }^{2}$ Department of Pediatrics and Medical Genetics, Medical Faculty - Plovdiv, 15A Vassil \\ Aprilov St., 4000 Plovdiv, Bulgaria \\ ${ }^{3}$ University Hospital "St. George" Plovdiv, 66 Peshtersko Shuse Str., Plovdiv 4000, Bulgaria \\ ${ }^{4}$ State Phsychiatry Hospital Pazardzhik, 28 Bolnichna Str. Pazardzhik, Bulgaria \\ *Corresponding author
}

\begin{abstract}
A B S T R A C T
Keywords

Schizophrenia, MiRNAs, Stem-loop qRTPCR, Whole blood

Article Info

Accepted: 20 February 2016 Available Online: 10 March 2016

To date, the development of biomarkers of schizophrenia still remains a challenge. Recently, alterations in the expression of microRNAs (miRNAs) from peripheral blood, serum and post-mortem brain tissue have been linked to schizophrenia and other neurodevelopmental disorders. It is well known that miRNAs are expressed by various cell types and can modulate broad physiological functions. On this basis it is assumed that miRNAs could be useful predictive biomarkers for the diagnosis or prognosis of pathological conditions, like schizophrenia. In order to observe an association between miRNAs and schizophrenia, this study was designed to investigate expression profiling of miRNAs in peripheral blood. The aim of this study was to test whether whole blood miR-320 family members display differential expression profile in schizophrenia patients. The relative expression levels of miR-320 members were analyzed by stem-loop qRT-PCR assay in acohort of 30 participants diagnosed with schizophrenia and 25 age- and gendermatched general population controls. The results indicated that miR-320b, miR$320 \mathrm{c}$ and miR-320d were expressed substantially higher in schizophrenia patients than in control subjects, and miR-320a showed thegreatest change. Furthermore, a statistically significant difference of mR-320a level was revealed in schizophrenia subjects compared to healthy controls $(p=0,0076)$. Our results suggest that differentially expressed miR-320 family members might be involved in schizophrenia molecular pathways and blood-based miR-320 signature might be able to serve as potential prognostic biomarkers for schizophrenia. The results confirm that the blood-based miRNA profiling is a feasible way to identify biomarkers for schizophrenia.
\end{abstract}

\section{Introduction}

Schizophrenia is a severe chronic mental population worldwide. MicroRNA disorder, affecting approximately $1 \%$ of the 
small non-coding +RNAs that negatively regulate the expression of more than $60 \%$ ofprotein-coding genes (Friedman et al., 2009). The nervous systemhas the most extensive profile of miRNA expression of all human tissues. Approximately $70 \%$ of all known miRNAs are expressed in the brain, where many of them are neuron's specific miRNAs (Krichevsky et al., 2003). MiRNAs have received increased attention in genetic studies of schizophrenia, as some support the hypothesis that miRNAs play an important role in brain disorders (Ma et al., 2013; Müller et al., 2014; Kumar et al., 2013; Shi et al., 2012).

Several recent studies on postmortem cortices have identified numerous miRNAs that are differentially expressed in schizophrenia patients (Beveridge et al., 2010; Perkins et al., 2007). In a study of Chinese population, $\mathrm{Xu}$ et al., described a potentially functional variant ss178077483 located in the pre-mir-30e that affected premiR-30e and was associated with schizophrenia (Xu et al., 2010). Even though the samples were obtained from the peripheral blood, the findings of $\mathrm{Xu}$ et al. were comparable to the observations of Perkins et al, who detected an increase in the expression level of $\mathrm{miR}-30 \mathrm{e}$ in the prefrontal cortex of patients with schizophrenia.

It is not surprising that investigation of miRNA expression in blood and postmortem brain tissue has revealed differences between schizophrenia patients and control group (Mellios and Sur., 2012; Beveridge et al., 2012). Several miRNAs, such as miR124, miR-132, miR-134, and miR-137 have been implicated in schizophrenia (Mellios and Sur., 2012).

In a recent work Gardiner et al., 2011 and Lai et al., 2011 speculated that schizophrenia-associated miRNA expression signatures may also be detected in nonneuronal tissue such as whole blood. Gardiner et al. investigated the expression profile of miRNA in peripheral blood mononuclear cells (PBMCs) of 112 patients with schizophrenia and 76 non-psychiatric controls (Gardiner et al., 2011). They identified 83 miRNAs that were significantly downregulated in the schizophrenia group, including a large subgroup of miRNAs transcribed from a single imprinted locus at the maternally expressed DLK1-DIO3 region on chromosome 14q32. In addition, similarly toGardiner et al. Lai and co-workers identified a specific profile of seven miRNAs in an initial cohort of 30 patients with schizophrenia and 30 controls. The specific signature detected,included upregulated miR-564, miR-34a, miR-449a, miR-548d, miR-652 and miR-572, and down regulated miR-432 (Lai et al., 2011). Results from that particular study were subsequently validated in an extended cohort of 60 schizophrenia patients and 30 controls. Autors carried out a miRNA expression profiling of PBMCs andfound a group of dysregulated miRNAs in schizophrenia patients. Lai et al. had also shown that the miRNA expression signatures observed in PBMCs in schizophrenia patients may have the potential to serve as predictive biomarkers of schizophrenia.

In the human genome there are eight annotated miR-320 genes encoding five mature miRNA variants including miR-320a (Chr 8), miR-320b (Chr 1), miR-320c (Chr 18), miR-320e (Chr 19), miR-320d-1 (Chr 13) and miR-320d-2 (Chr X), last two with the same mature miRNA sequences. The current study aim to elucidate the expression profile of particular miR-320 family members through qRT-PCR assay. 


\section{Materials and Methods}

\section{Participants and Clinical Assessment}

This study design and the Inform Concent Form (ICF) were approved by the Ethics Committee of Medical University of Plovdiv. The Institutional Review Board approved the use of the samples for this study. Thirty schizophrenia patients were recruited from State Psychiatric Hospital Pazardzhik, Bulgaria after obtaining written informed consent.

Routine psychiatric examination and wide medical history were done by a certified psychiatrist to evaluate the diagnosis of paranoid schizophrenia, using Diagnostic and Statistical Manual of Mental Disorders fourth edition Text Revision (DSM IV TR) criteria. In order to exclude any other mental disorder of the study and control groups the Mini-International Neuropsychiatric Interview version 5.0 was also done. The sample population included 32 participants diagnosed with schizophrenia and 24 ageand gender-matched general population controls. Main inclusion criteria was that the participants did not receive any medication before blood sampling for at least 2 weeks. Persons with other medical illness or psychoactive substances (with exception of nicotine) users were excluded from the cohorts of interest.

\section{Blood Collection and RNA Extraction}

The peripheral blood samples $(2.5 \mathrm{ml})$ for each participant (schizophrenia and healthy controls) were collected into PAX gene blood RNA tubes (PreAnalytiX). Total RNA was isolated using PAXgene blood miRNA kit (PreAnalytiX), according to the manufacturer's protocol. Assessment of A260/A280 ratios revealed that all analyzed RNA samples are with sufficient quality for
qRT-PCR analysis (1.93 - 2.10). RNA quality and purity were analyzed by Epoch Micro-Volume Spectrophotometer System (BioTek). RNA integrity of all samples (schizophrenia and healthy controls) was also checked by agarose gel electrophoresis.

\section{Confirmation of Differentially Expressed miR-320 Family Members in Whole Blood by Stem-Loop qRT-PCR Analysis}

Quantitative RT-PCR was used to measure the expression of individual miR-320 family members. MicroRNA specific cDNA was synthesized from equal mass inputs of $1 \mu \mathrm{g}$ of total RNA using RevertAid First Strand cDNA Synthesis Kit (Thermo Fisher Scientific) using miRNA-specific stem-loop primers. Five microliters of each miRNA specific cDNA were subjected to preamplification prior to the real-time PCR step with Long Range PCR Kit (Qiagen) to potentially enhance sensitivity of the assay. Copy DNA for the protein coding gene was synthesized from total RNA with oligo (dT) 18 primer using Revert Aid First Strand cDNA Synthesis Kit according to the assay protocol (Thermo Fisher Scientific, Waltham, MA USA) using $1 \mu \mathrm{g}$ of total RNA samples. qRT-PCR was performed using Maxima SYBR Green qPCR Master Mix (Thermo Fisher Scientific) with ABI PRISM 7500 (Applied Biosystems).

The qRT-PCR primers used are listed in Table.1. The expression levels of miRNAs for qRT-PCR were normalized to the level of human B Actin, and relative quantification (RQ) was calculated utilizing the $2^{-\Delta \Delta C t}$ method. PCR amplification of the specific products corresponding to B Actin andeach specific miR-320 amplicons was confirmed by monitoring the dissociation curve (Melting curve analisys) and by agarose gel electrophoresis. 
Table 1. MiR-320 specific qRT- PCR primer sets

\begin{tabular}{lll}
\hline MiRNAs & \multicolumn{1}{c}{ Primer sequence 5'- $\mathbf{3}$} \\
\hline miR-320b SL & CTCAACTGGTGTCGTGGAGTCGGCAATTCAGTTGAGTTGCCCTC \\
miR-320b For & ACACTCCAGCTGGGAAAAGCTGGGTTGAGA \\
miR-320a SL & CTCAACTGGTGTCGTGGAGTCGGCAATTCAGTTGAGTCGCCCTC \\
miR-320a $\quad$ For & ACACTCCAGCTGGGAAAAGCTGGGTTCAGA \\
miR-320c SL & CTCAACTGGTGTCGTGGAGTCGGCAATTCAGTTGAGACCCTCTC \\
miR-320c For & ACACTCCAGCTGGGAAAAGCAGGGTTGA \\
miR-320d SL & CTCAACTGGTGTCGTGGAGTCGGCAATTCAGTTGAGTCCTCTCA \\
miR-320d For & ACACTCCAGCTGGGAAAAGCTGGGTTG \\
Universal Rev & GTCGGCAATTCAGTTGAG \\
B ActinFor & AGTGTGACGTGGACATCCGCA \\
B ActinRev & GCCAGGGCAGTGATCTCCTTCT \\
\hline
\end{tabular}

\section{Statistical Analysis}

All statistical calculations were performed using the Statistical Package for the Social Sciences software, version 20.0 (SPSS Inc., Chicago, IL, USA). Analysis of variance (ANOVA) $t$-test of $\mathrm{Ct}$ was used to examine differences in expression levels of miR-320 members across healthy controls and schizophrenia subjects, considering Kolmogorov-Smirnov criteria for normal distribution and Fisher-Snedecor distribution. A Spearman correlation analysis was also done for searching relation between expression levels and demographic characteristics of the tested individuals. The expression level of each miRNA was quantified by its normalized threshold cycle number $\Delta \mathrm{Ct}$, in which $\Delta \mathrm{Ct}=[\mathrm{Ct}(\mathrm{miRNA})]-$ [Ct (B Actin)], and the relative expression level was calculated as $2^{-(\Delta \Delta \mathrm{Ct})}$, which is commonly used in miRNAs profiling studies (Schmittgen et al., 2008). Logistic Regression analysis was done to determine predicted probabilities of miRNA 320 expression levels. Subsequent ROC (receiver operating characteristic) curve analyses was performed so specificity and sensitivity can be obtained.

\section{Results and Discussion}

MiRNA expression was analyzed in peripheral blood of 32 patients with schizophrenia and 24 non-psychiatric controls using miR-320 family specific stem-loop qRT-PCR assay. The PCR amplification of the specific products corresponding to $\mathrm{B}$ actin and miR-320 amplicons was confirmed by monitoring the dissociation curve (melting curve analysis). Our analytical approach allows the quantification of specific miR-320 family members as alterations in expression of individual miRNAs in schizophrenia. While qRT-PCR validation showed that all of the analysed miRNAs were up-regulated (miR320b, miR-320c, miR-320d), only miR-320a showed statistically significant difference $(p$ $=0,0114)$ (Figure 2). Two-dimensional hierarchical clustering of differentially expressed miR-320 genes between schizophrenia and control group subjects as observed bystem-loop qRT-PCR are shown in Figure 1.Alterati ons in miRNA levels are presented as the fold change in gene expression normalized to an endogenous reference gene and relative to the healthy 
control patients. The results are shown by analyzing differences between schizophrenia and control groups, (Figure 2) and analyzing individual differencesfor each miR-320 family members between groups (Figure1). To evaluate the potential of the miR-320 family members as biomarkers of schizophrenia, we performed ROC analysis of the data from the qRT-PCR results. A ROC curve was done and AUC (area under the ROC curve) was calculated. ROC analysis was subsequently used to assess the diagnostic sensitivity and the specificity (Figure3).

Figure.1 Two-dimensional hierarchical clustering of differentially expressed miR-320 genes between schizophrenia and control group subjects. Each line represents a gene, and each column is a sample. The expression signal intensities are shown in red and blue, indicating high and low expression, respectively. Names of the miR-320 transcripts are indicated at right. Comparisons of statistically significant differentially expressed miR-320a with other members miR-320d, miR-320b and miR-320c in panels (B1, B2, B3) respectively
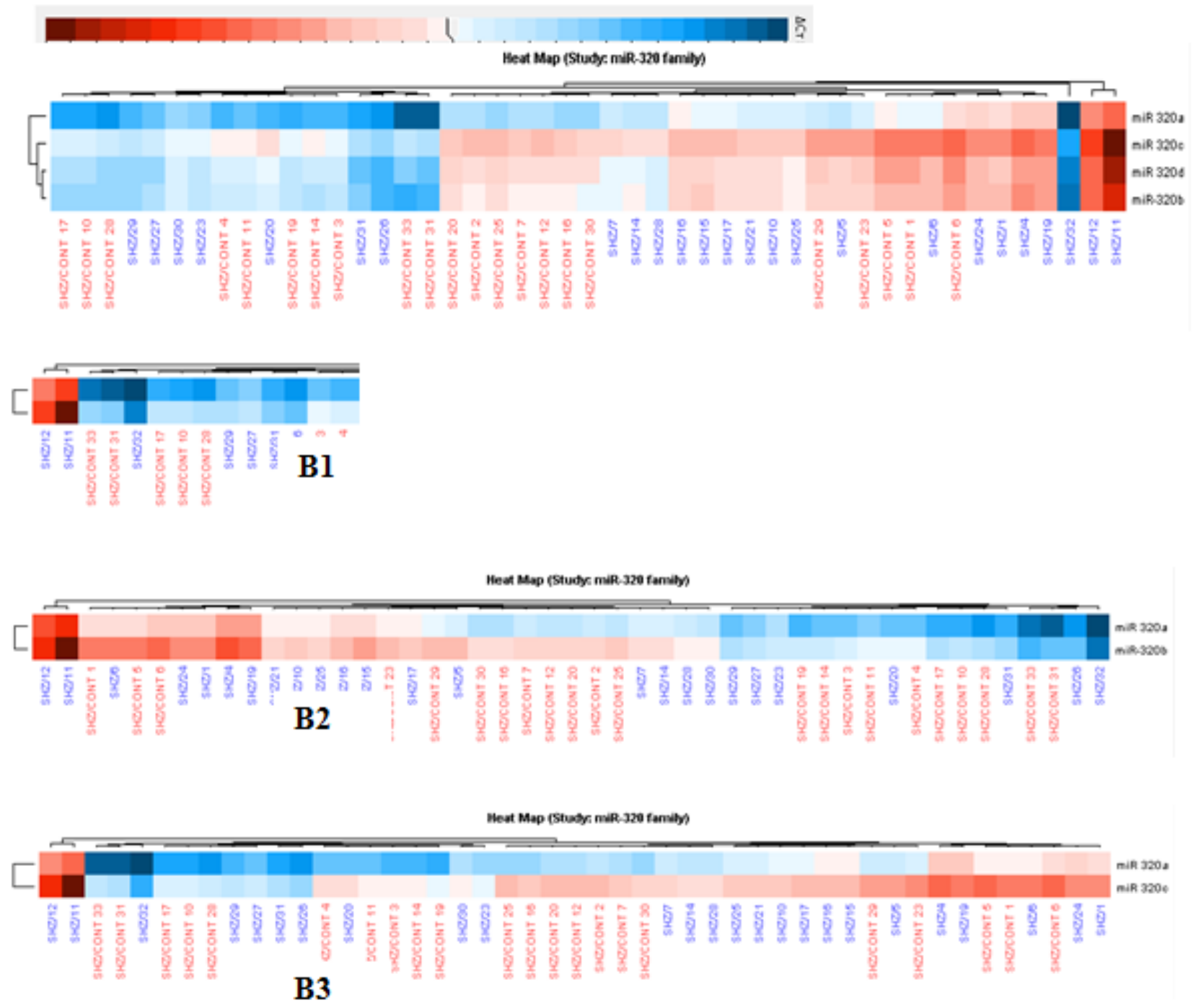
Figure.2 Schizophrenia associated miR-320 up regulation in peripheral blood. Quantitative realtime RT-PCR reaction analysis of miRNA genes in schizophrenia versus control group. Expression levels (left diagram) of miR-320a, miR-320c $(\log 2(\mathrm{RQ})=0.35)$, miR-320d $(\log 2$ $(\mathrm{RQ})=0.35)$ and miR-320b $(\log 2(\mathrm{RQ})=0.47)$, respectively. Bars represent fold change in expression. Box plot of miR-320a expression (right diagram), indicating a 0,79 fold increase for miR-320a in cases relative to controls
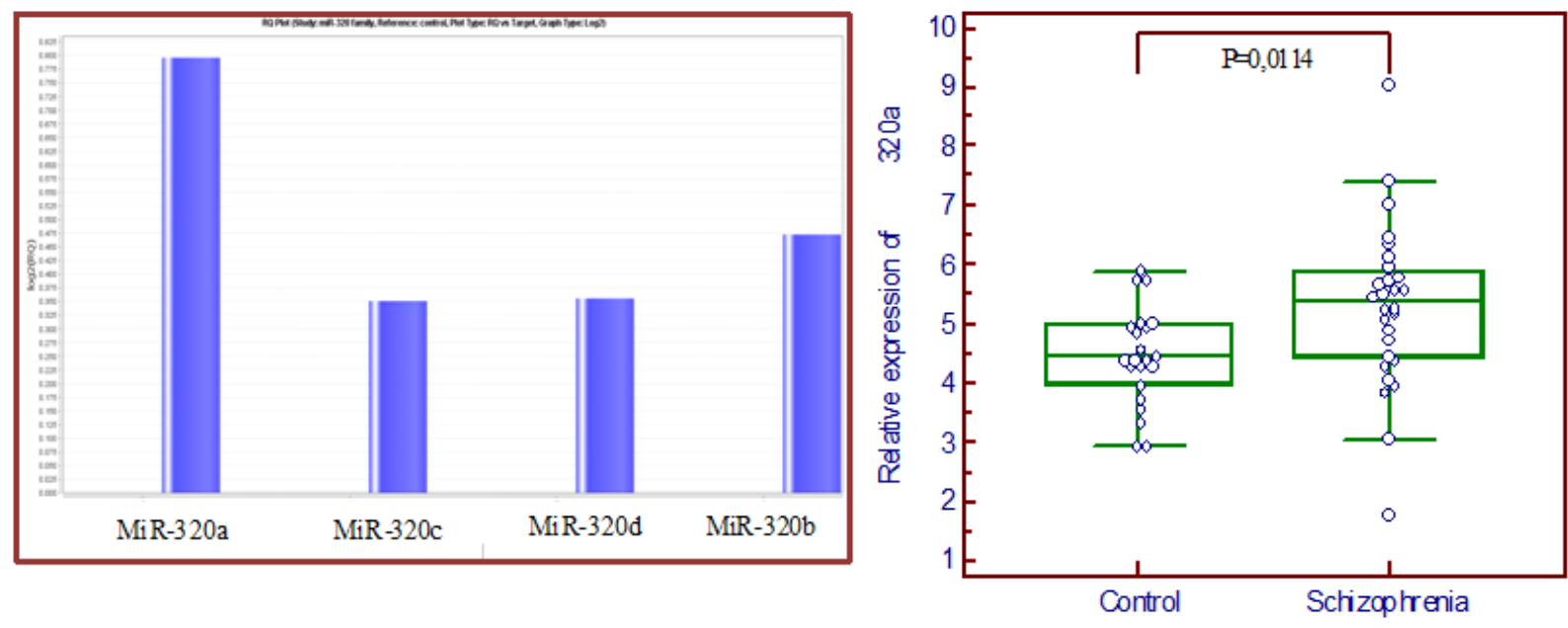

Figure.3 Performance of the miR-320 prediction model. The ROC for miR-320 gene expression was performed to evaluate the prediction accuracy. The dotted diagonal line represents random classification accuracy (AUC 0.5). ROC curves were drawn for miR-320a, miR-320b, miR-320c and miR-320d which yielded 0,722, 0,574, 0,529,0,524 as AUC values, respectively.

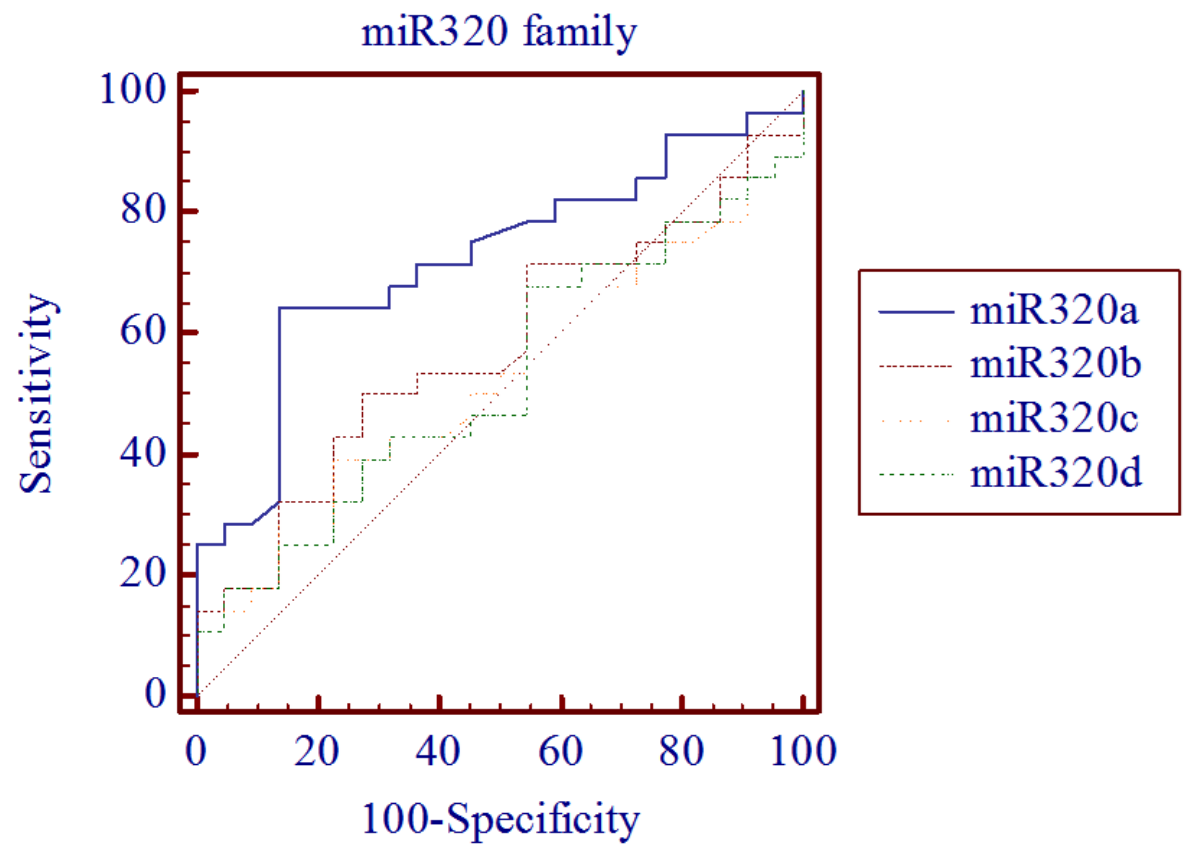


Figure.4 Combined ROC curve of the differentially expressed miR-320 family members (miR320a, miR-320b, miR-320c and miR-320d). The diagonal line represents random classification accuracy (AUC 0.5). Diagnostic sensitivity of combined classifiers were $85,7 \%$ with the corresponding specificity of $95,5 \%$

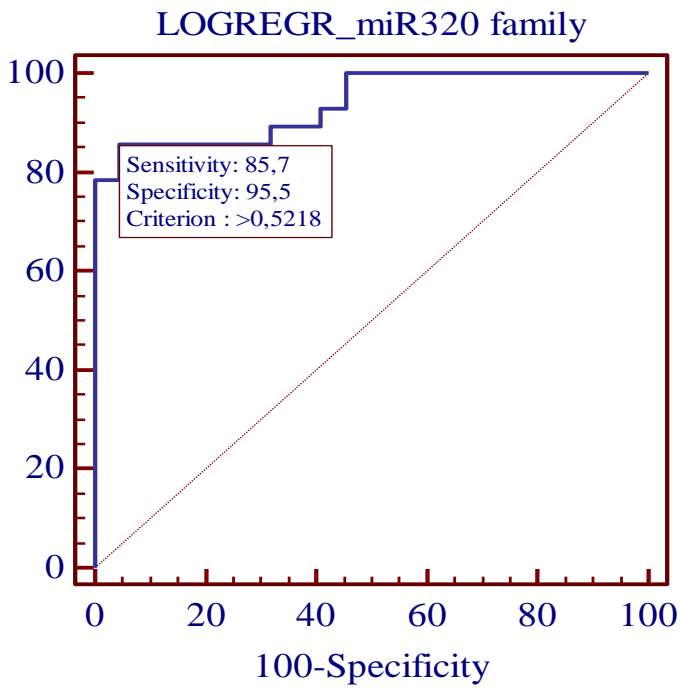

Figure.5 Sex-dependent variations in the miR-320a expression pattern between analyzed groups, according to Kruskal-Wallis test

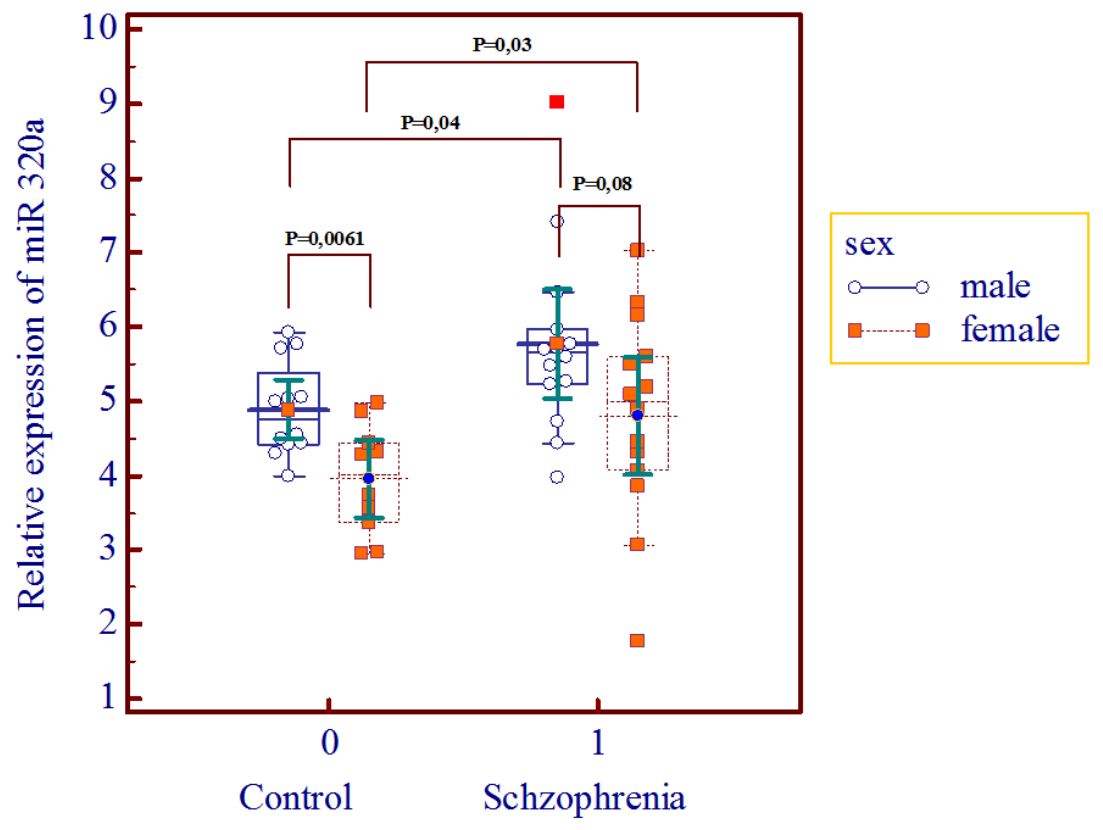


Figure.6 Relative miRNAs levels are shown for miR-320b, miR-320d, miR-320c, and miR-320a that demonstrate differential expression levels when comparing 32 individuals with schizophrenia and 24 healthy controls. miRNA levels were normalized to the endogenous reference gene relative to the healthy control patients. Whole blood samples were analyzed for the expression of selected miRNAs by stem-loop RT-qPCR. Data represent the expression fold changes (RQ) relative to healthy controls
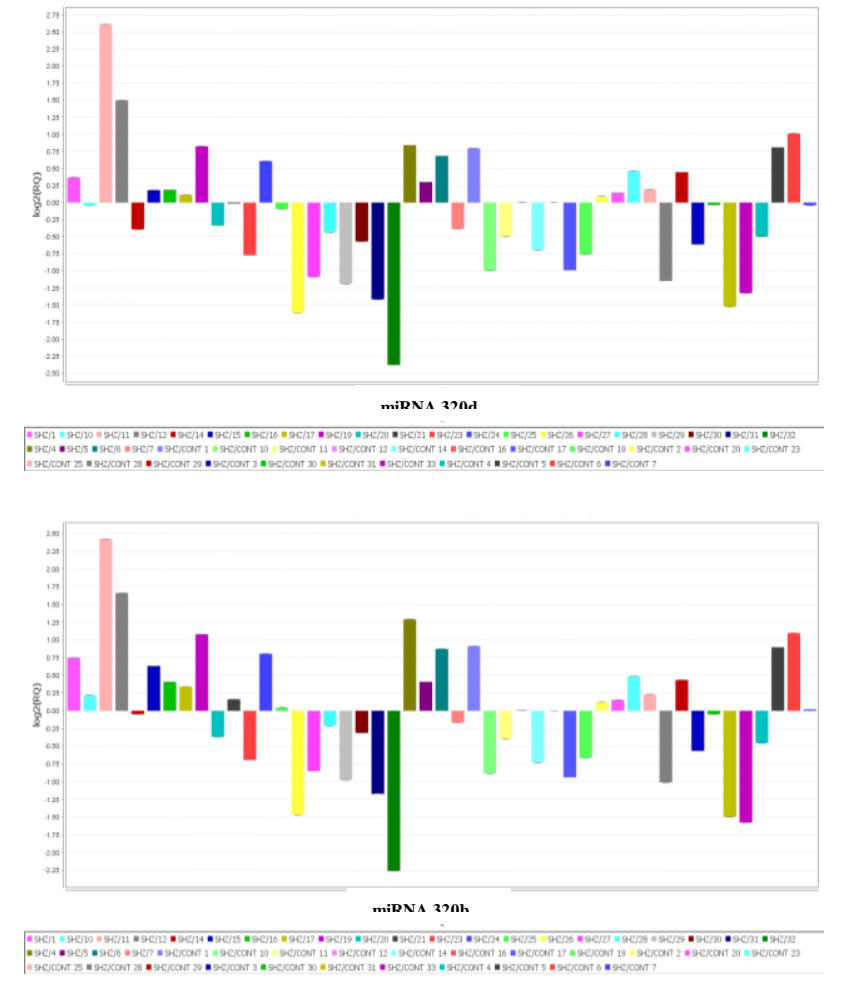

Diagnostic sensitivities of miR-320a, miR320b, miR-320c and miR-320d, for schizophrenia were $64,3 \%, 50,0 \%, 39,3 \%$ and $67,9 \%$, respectively. The corresponding specificities were $86,4 \%, 72,7 \%, 77,3 \%$ and $45,5 \%$, and ROC curve areas $(95 \% \mathrm{CI})$ were $0,722(0,577$ - 0,839\%), 0,574 (0,426 $0,713 \%), 0,529(0,383-0,672 \%)$ and 0,524 $(0,378-0,667 \%)$, respectively. Additionally, a combined ROC curve of the differentially expressed miR-320 family members was done. Diagnostic sensitivity and specificities of combined classifiers are shown in Figure 4. We also analysed sexdependent variations in the miR-320a expression pattern between analyzed groups. As shown in Figure 5, no significant
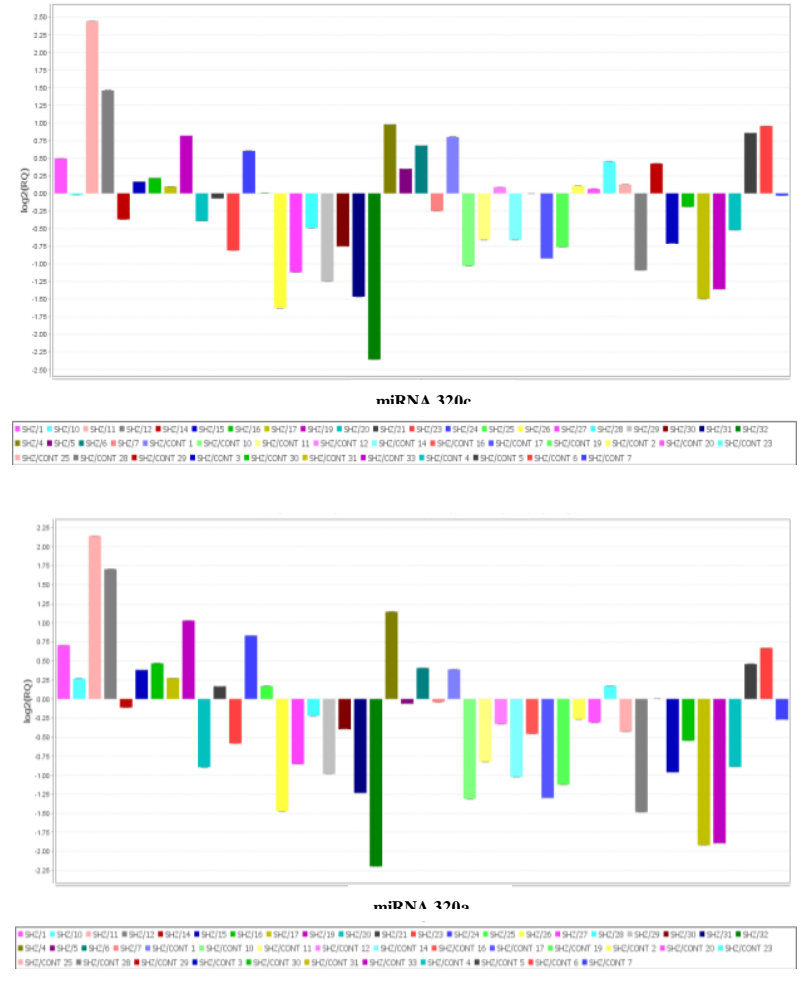

difference of miR-320 a expression level was found between male and female schizophrenia patients $(\mathrm{P}=0.08)$. However, there was significant difference between male and female healthy controls in miR$320 \mathrm{a}$ expression $(\mathrm{P}=0.0061)$. There was significant difference in $\operatorname{miR}-320$ a expression level between female and male schizophrenia patients compared to healthy controls $\quad(\mathrm{P}=0.03) \quad$ and $\quad(\mathrm{P}=0.04)$, respectively.

Multiple studies have shown that defective function and neuronal plasticity in neurodevelopmental disorders may have resulted from impaired miRNA mediated post transcriptional regulation. Numerous 
expression studies have described dysregulation of members of the miR-320 family in patients with various neuropsychological disorders. Lopez et al., conducted miRNA expression profiling in postmortem brain samples (Brodman area 44) of individuals with major depressive disorder, committed suicide, using a TaqMan qRT-PCR absolute quantitation

The use of postmortem brain tissue has several limitations, including availability, potential for long delay between death and sample collection, during which rapid Cellular and molecular changes can occur, the possible lack of medication history and the difficulty inseparating the effect of disease from other confounding factors (Popova et al., 2008). Since brain tissue is not readily accessible, a new focus in research on biomarkers is blood-based expression profiling of miRNAs. In order to determine the expression profile of miRNAsperipheral blood leukocytes in people, affected by Parkinson disease (PD), Soreq et al., conducted SOLiD high throughput sequencing on small RNAs from peripheral blood leukocytes of PD patients followed by alignment to miRBase. (Soreq et al., 2013). In this particular study mir320a, mir-320b and mir-320c show statistically significant downregulation in the group of PDaffected patients compared with healthy controls.

Tabares-Seisdedos et al., reviewed the short arm of chromosome 8 as a potential site for $\mathrm{CNV}$ alterations, closely related to different neurodevelopment disorders such as depression, schizophrenia and autistic spectrum disorder (ASD). Among the 484 genes located in this region 7 are microRNA genes, including miR-320. Based on evidence obtained in molecular genetics and cerebral developmental studies, authors suggest that alterations in FGF17, mir-124-1 and mir-320 should be considered to endow standard curve approach. In this study authors show that mir-320c displays statistically significant upregulation in suicide committers compared to controls. They also found significant negative correlation between miR-320c levels and the levels of the SAT1 gene, whose expression is referred to be lower in brain tissues of suicide completers (Lopez et al., 2014) susceptibility to mental illness (TabaresSeisdedos et al., 2009). The authors, however do not specify the exact member of the miR-320 family in their review. However, assuming that miR-320a gene is the only one in the family located on the chromosome 8 , we deduce that this is the specific micro RNA gene the authors have refered to.

In this study we have performed, miRNA specific stem loop qRT-PCR assay in order to identify microRNAs involved in schizophrenia. Despite the fact that we do not know whether the aberrant expression of the miRNAs were also present in the patients' CNS, the majority of them are detectable in the brain according tothe literature (Hsu et al., 2008; Wheeler et al., 2006). The changes in the posttranscriptional regulatory environment of peripheral blood in patients with schizophrenia not only have implications for the pathophysiology of the disorder (Perkins et al., 2005; Beveridge et al., 2010) but also provide a suitable source of non-invasive biomarkers for schizophrenia. The study of the differential expression of miRNA molecules is very important since dysregulation in the expression levels of specific miR-320 members are expected to influence the transcription/translation of a number of protein-encoding genes. We found that miR-320b, miR-320c, miR-320d are upregulated in schizophrenia and miR-320aas the most up-regulated.To our knowledge, this is the first study to show evidence from whole blood where the expression of miR- 
$320 \mathrm{a}$ is dysregulated in subjects with schizophrenia.

\section{Acknowledgement}

The authors are most grateful to the probands for its collaboration. This study was supported by MU - Plovdiv Project № $\mathrm{HO}-21 / 2014$.

\section{References}

Beveridge, N.J, Cairns, M.J.2012. Micro RNA dysregulation in schizophrenia. Neurobiol Dis. 46:263-271.

Beveridge, N.J., Gardiner, E., Carroll, A.P., Tooney, P.A., Cairns, M.J. 2010. Schizophrenia is associated with an increase in cortical microRNA biogenesis. Mol Psychiatry15:117689.

Friedman, R.C., Farh, K.K., Burge, C.B., Bartel, D.P.2009. Most mammalian mRNAs are conserved targets of microRNAs. Genome Research 19:92e105.

Gardiner, E., Beveridge, N.J., Wu, J.Q., Carr, V., Scott, R.J., Tooney, P.A., Cairns, M.J. 2011. Imprinted DLK1DIO3 region of $14 q 32$ defines a schizophrenia-associated miRNA signature in peripheral blood mononuclear cells. Mol Psychiatry 17:827-40.

Hsu, S.D., Chu, C.H., Tsou, A.P., Chen, S.J., Chen, H.C., Hsu, P.W., Wong, Y.H., Chen, Y.H., Chen, G.H., Huang, H.D. 2008. miRNAMap 2.0: genomic maps of microRNAs in metazoan genomes. Nucleic Acids Res 36: D165-169.

Krichevsky, A.M., King K.S., Donahue, C.P., Khrapko K., Kosik, K.S. 2003.A microRNA array reveals extensive regulation of microRNAs during brain development. RNA9:1274e81.
Kumar, P., Dezso, Z., MacKenzie, C., Oestreicher, J., Agoulnik, S., Byrne, M., Bernier, F., Yanagimachi, M., Aoshima, K., Oda, Y. 2013. Circulating miRNA biomarkers for Alzheimer's disease. PLoS One 8: e69807

Lai, C.Y., Yu, S.L., Hsieh, M.H., Chen, C.H., Chen, H.Y., Wen, C.C., Huang, Y.H., Hsiao, P.C., Hsiao, C.K., Liu, C.M., Yang, P.C., Hwu, H.G., Chen, W.J. 2011. MicroRNA expression aberration as potential peripheral blood biomarkers for schizophrenia. PLoS One. 6:e21635.

Lopez, J.P.,Fiori, L.M.,Gross, J.A.,Labonte, B.,Yerko, V.,Mechawar N.,Turecki, G. 2014. Regulatory role of miRNAs in polyamine gene expression in the prefrontal cortex of depressed suicide completers.

Int $\mathbf{J}$

Neuropsychopharmacol17(1):23-32.

Ma, L., Wei, L., Wu, F., Hu, Z., Liu, Z., Yuan, W.2013. Advances with microRNAs in Parkinson's disease research. Drug Des Devel Ther 7: 1103-1013

Mellios, N., Sur, M.2012. The emerging role of microRNAs in schizophrenia and autism spectrum disorders. Front Psychiatry. 3:39.

Müller, M., Kuiperij, H.B., Claassen, J.A., Küsters, B., Verbeek, M.M.2014. MicroRNAs in Alzheimer's disease: differential expression in hippocampus and cell-free cerebrospinal fluid. Neurobiol Aging 35 :152-158

Perkins, D.O., Jeffries, C., Sullivan, P. 2005. Expanding the 'central dogma': the regulatory role of nonprotein coding genes and implications for the genetic liability to schizophrenia. Mol Psychiatry 10: 69-78.

Perkins, D.O., Jeffries, C.D., Jarskog, L.F., Thomson, J.M., Woods, K., Newman, M.A., Parker, J.S., Jin, J., Hammond, 
S.M. 2007. microRNA expression in the prefrontal cortex of individuals with schizophrenia and schizoaffective disorder. Genome Biol 8:R27.

Popova, T., Mennerich, D., Weith, A., Quast, K. 2008. Effect of RNA quality on transcript intensity levels in microarray analysis of humanpostmortem brain tissues. BMC Genomics. 9:91.

Schmittgen, T.D., Lee, E.J., Jiang, J., Sarkar, A., Yang, L., Elton, T.S., Chen, C. 2008. Real-time PCRquantification of precursor and mature microRNA. Methods 44: 31-38.

Soreq, L., Salomonis, N.,Bronstein, M., Greenberg, D.S.,Israel, Z.,Bergman, H.,Soreq H. 2013. Small RNA sequencing-microarray analyses in Parkinson leukocytes reveal deep brain stimulation-induced splicing changes that classify brain region transcriptomes.Front Mol Neurosci6: 10.doi:10.3389/fnmol.2013.00010.
Shi, W., Du, J.,Qi. Y., Liang, G., Wang, T., Li, S., Xie, S., Zeshan, B., Xiao, Z. 2012. Aberrant expression of serum miRNAs in schizophrenia. J Psychiatr Res 46: 198-204

Tabares-Seisdedos, R., Rubenstein, J.L. 2009. Chromosome $8 \mathrm{p}$ as a potential hub for developmental neuropsychiatric disorders: implications for schizophrenia, autism and cancer. Mol. Psychiatry 14(6):563-89.

Wheeler, G., Ntounia-Fousara, S., Granda, B., Rathjen, T., Dalmay, T. 2006. Identification of new central nervous system specific mouse microRNAs. FEBS Lett 580: 2195-2200.

Xu, Y., Li, F., Zhang, B., Zhang, K., Zhang, F., Huang, X., Sun, N., Ren, Y., Sui, M., Liu, P. 2010.microRNAs and target site screening reveals a premicroRNA-30e variant associated with schizophrenia. Schizophr Res 119: 219-227.

\section{How to cite this article:}

Tihomir Iliev Vachev, Nikolay Todorov Popov, Vili Krasteva Stoyanova, Hristo Yordanov Ivanov and Danail Savov Minchev. 2016. Alterations of miR-320 Family Members as a Novel Diagnostic Biomarkers in Peripheral Blood of Schizophrenia Patients. Int.J.Curr.Microbiol.App.Sci. 5(3): 865-875. doi: http://dx.doi.org/10.20546/ijcmas.2016.503.100 